\title{
Comparison of antenna diversity schemes
C.
}

${ }^{1}$ K.U.Leuven, Kard. Mercierlaan, 94; B-3001 Heverlee; Belgium; 甶+32/16/321113; Fax:

+32/16/321986; vanlil@esat.kuleuven.ac.be; ${ }^{2}$ Fund for Scientific Research (Flanders)

${ }^{3}$ Univ. of Vigo, Spain.

\section{Summary}

Even if the foundations for diversity studies have been laid out long ago [1], it took until both fast computers and efficient computation schemes [2] were available before simulations of complex scenarios was possible. This paper compares 4 kinds of simple diversity schemes for an indoor scenario:

a. The first uses polarisation diversity and a dual polarised antenna. Its advantage is that the antenna does not take up more space than a single antenna.

b. The second combines the signals of 2 antennas, where the distance between the two antennas was chosen such that the correlation was smaller than 0.5 (in this case one wavelength).

c. The third combines antenna and polarisation diversity.

d. The fourth uses 3 antennas, not placed like in [1] on an equilateral triangle, but forming a simple equidistant linear array with an inter-element spacing of one wavelength.

A few representative but still simple test cases have been computed. The first was a reference room

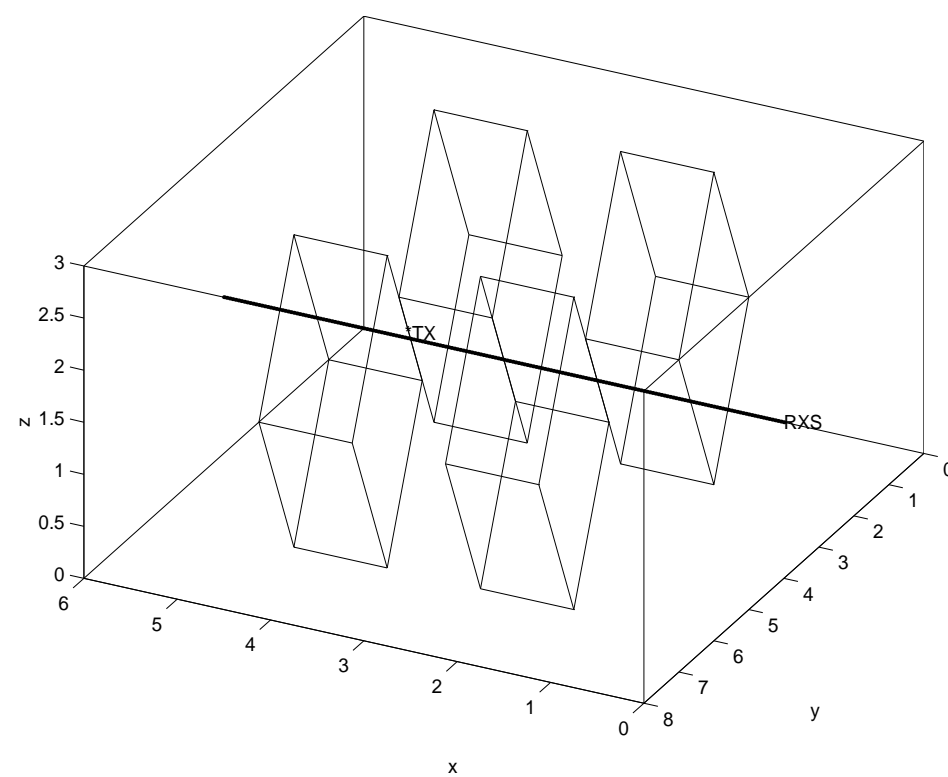
taken from the literature [3]. Because the level of the different components of the fields were significantly different due to the perfectly aligned vertical walls of the objects, and this is not encountered in practical measurements [4], we can better illustrate the advantages of diversity schemes by using a more futuristic looking chamber (Fig. 1). The position of the transmitter is indicated Fig. 1, and the receiver moves along a line where the field is sampled with a high resolution (961 points $=1$ point per $\lambda / 20$ ) partly in LOS conditions and partly in NLOS conditions.

Fig. 1: Chamber with oblique objects.

Field computations were made with a GO procedure and not with the improved model that we have developed [2], because this will not change significantly the statistics and hence our conclusions. We can also see from the fields (Fig. 2) that the central part is in LOS condition, and that the other polarisations are relatively small there, while the other parts are in NLOS conditions and do have significant cross-polar levels.

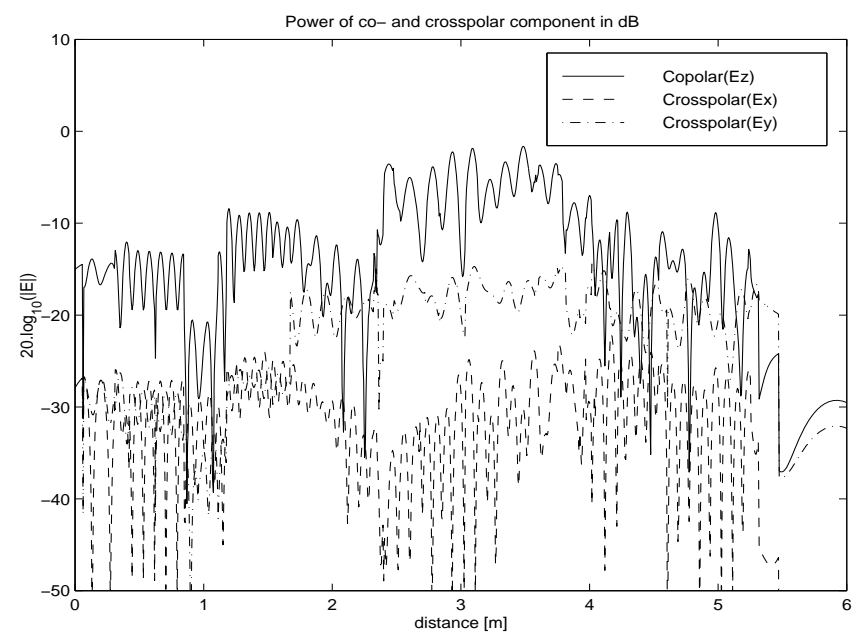

Fig. 2: The 3 components of the field along the trajectory. 


\section{Results and interpretation}

5 combining methods were considered:

1. The first combines all elements in phase in a power combiner. This gives either a broadside array for the antenna combining methods or a linearly polarised antenna for the polarisation combining methods. 2. The second combines all elements with a $90^{\circ}$ phase shift in a power combiner. This tilts the beam in case of an array, or makes a circularly polarised antenna in the polarisation combining case.

3. The third chooses the largest signal.

4. The fourth adds the decoded signals together (MRC). This corresponds with a factor of $\sqrt{ } \mathrm{M}$ with respect to a mere switching between the antennas ( $M=2$ in cases $a, b$ and c; $M=3$ for case $d$ ).

5 . The fifth combines the powers of the signals.

Some significant statistical parameters are summarised in Table 1. Not only the increase of the mean levels, but specially the minimum levels show that the power combing method is the best. The next rows show the improvement in signal level obtained for respectively $99 \%, 90 \%$ en $80 \%$ of the cases. (or that is not obtained in 1\%, 10\% and 20\% of the cases). A graphical representation of the CDF' $s$ is seen for the case of polarisation diversity in Fig. 3. The 2 RF combining methods even give worse results than the single-antenna case. The vertical scale used is the same as in [5], and has the advantage that a Rayleigh CDF is represented as a straight line. As a check, both a Rayleigh and a Rice CDF fitted to the signal of the reference antenna (Copolar) is also represented on the same plot. The match to the curve of antenna 1 is not perfect because the signal has both LOS (Rice) and NLOS (Rayleigh) parts. The number of points is sufficient to allow comparisons on levels above $99.9 \%$.

Table 1: Comparison of the signal levels of the polarisation combination methods ( $\mathrm{x}-\mathrm{\&} \mathrm{z}-$ components).

\begin{tabular}{|l|r|r|r|r|r|r|r|}
\hline & \multicolumn{1}{|c|}{ Vertical } & \multicolumn{1}{l}{ Horizontal } & \multicolumn{1}{l|}{ Linear } & \multicolumn{1}{l|}{ Circular } & \multicolumn{1}{l|}{ Maximum } & \multicolumn{1}{l|}{ Amplitude } & \multicolumn{1}{l|}{ Power } \\
\hline Mean & -12.2 & -21.4 & -15.5 & -13.9 & -12.0 & -12.6 & -11.5 \\
\hline$\sigma$ & -14.5 & -26.2 & -18.5 & -16.1 & -14.7 & -16.2 & -14.7 \\
\hline Min. & -40.5 & -62.0 & -62.6 & -43.7 & -37.1 & -34.4 & -34.4 \\
\hline Max. & -1.6 & -14.4 & -5.0 & -3.4 & -1.6 & -3.1 & -1.5 \\
\hline $99 \%$ & & & -22.0 & -0.3 & 0.4 & 3.0 & 3.0 \\
\hline $90 \%$ & & & -2.4 & -1.3 & 0.9 & 1.7 & 1.8 \\
\hline $80 \%$ & & & -2.2 & -0.8 & 2.4 & 2.5 & 3.4 \\
\hline
\end{tabular}
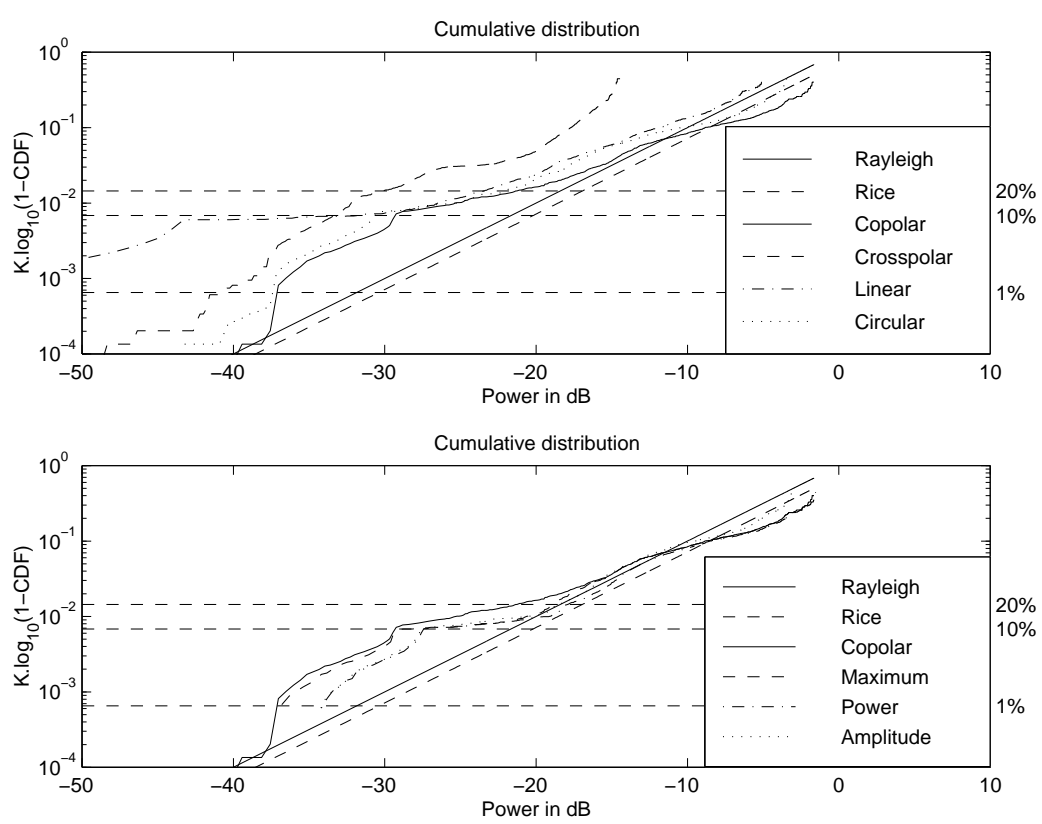

Fig. 3: Cumulative distribution functions of the polarisation diversity.

From the amplitude correlation plots (Fig. 4) one can see that the minimal separation for the 2-antenna case (where the correlation is less than 0.5 ) is a little bit more than 1 wavelength. This is the value that we have taken to apply the 5 diversity schemes. The corresponding CDF's are given in Fig. 5 . The same conclusions do apply: the power combination gives the best result, but the improvement is better than in the polarisation diversity case. 

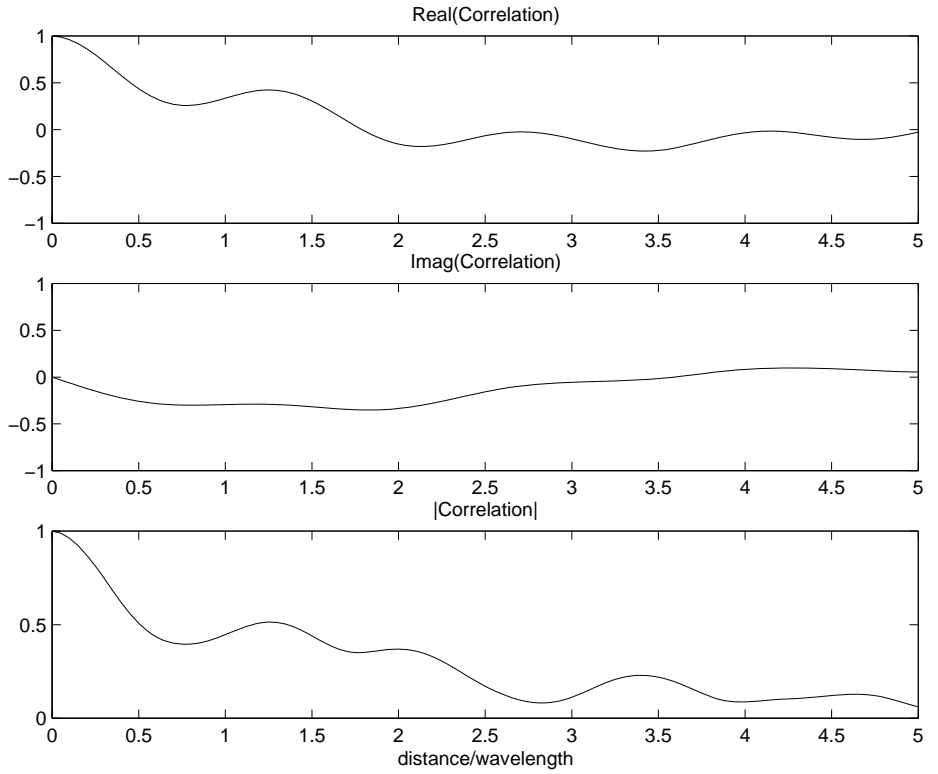

Fig. 4: Correlation plots for the 2-antenna case in function of the distance.
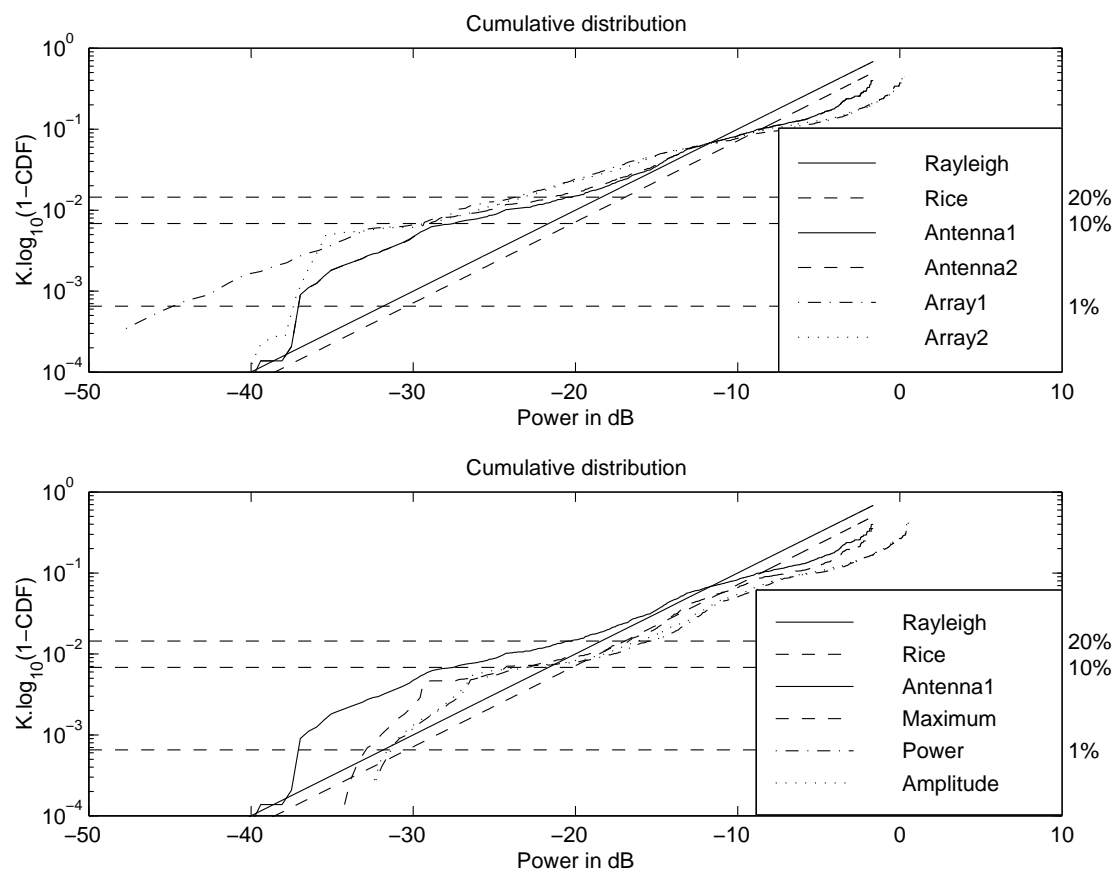

Fig. 5: Cumulative distribution functions of the polarisation diversity.

The third combining method might make use of both previous methods. The second antenna is perpendicular to the first, but at a distance from the first. The correlation plots (Fig. 6) learn us that the polarisation diversity case was already decorrelated, but the separation has also be taken 1 wavelength, like in the 2-antenna diversity case. The CDF's (Fig. 7) show us that the improvement is better than for the case of the polarisation diversity, but still not as good as the 2-antenna case. 

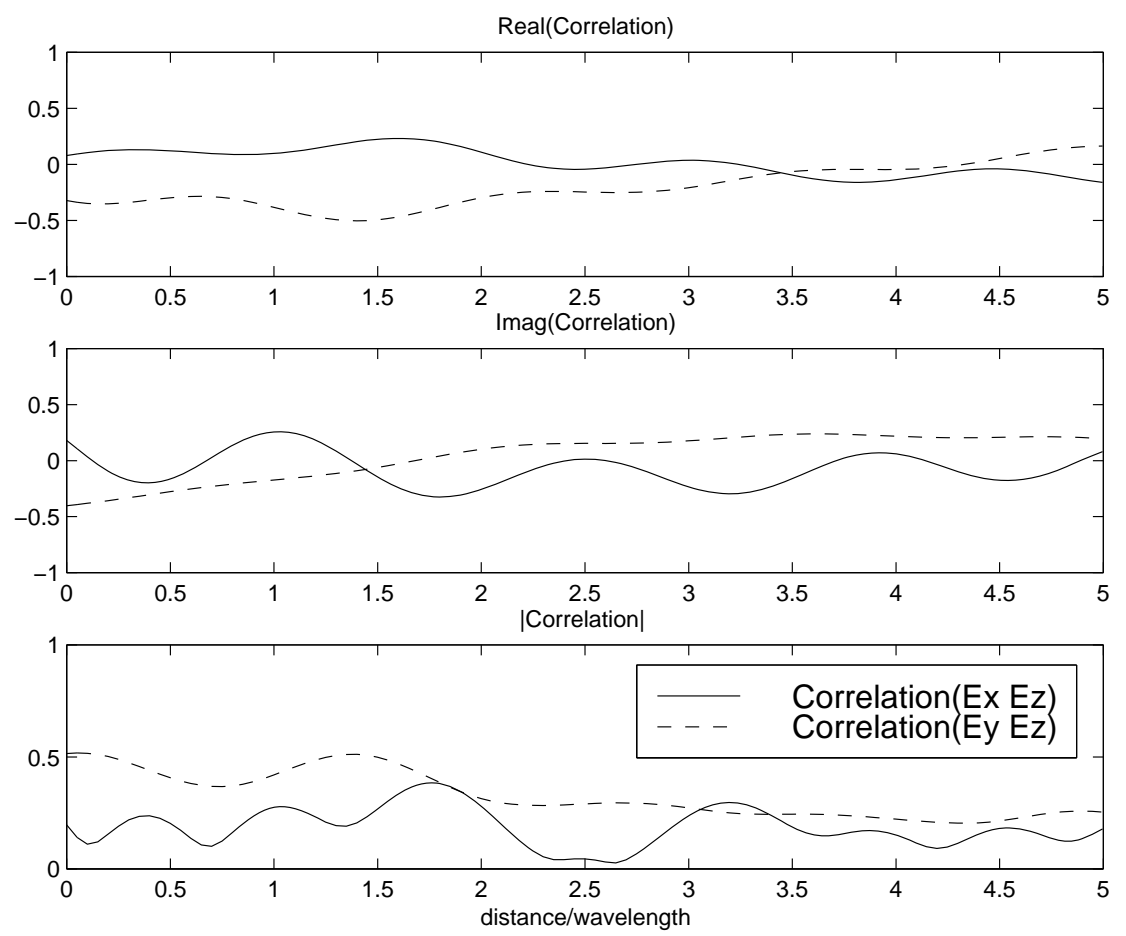

Fig. 6: Correlation plots for the antenna and polarisation diversity case in function of the distance.
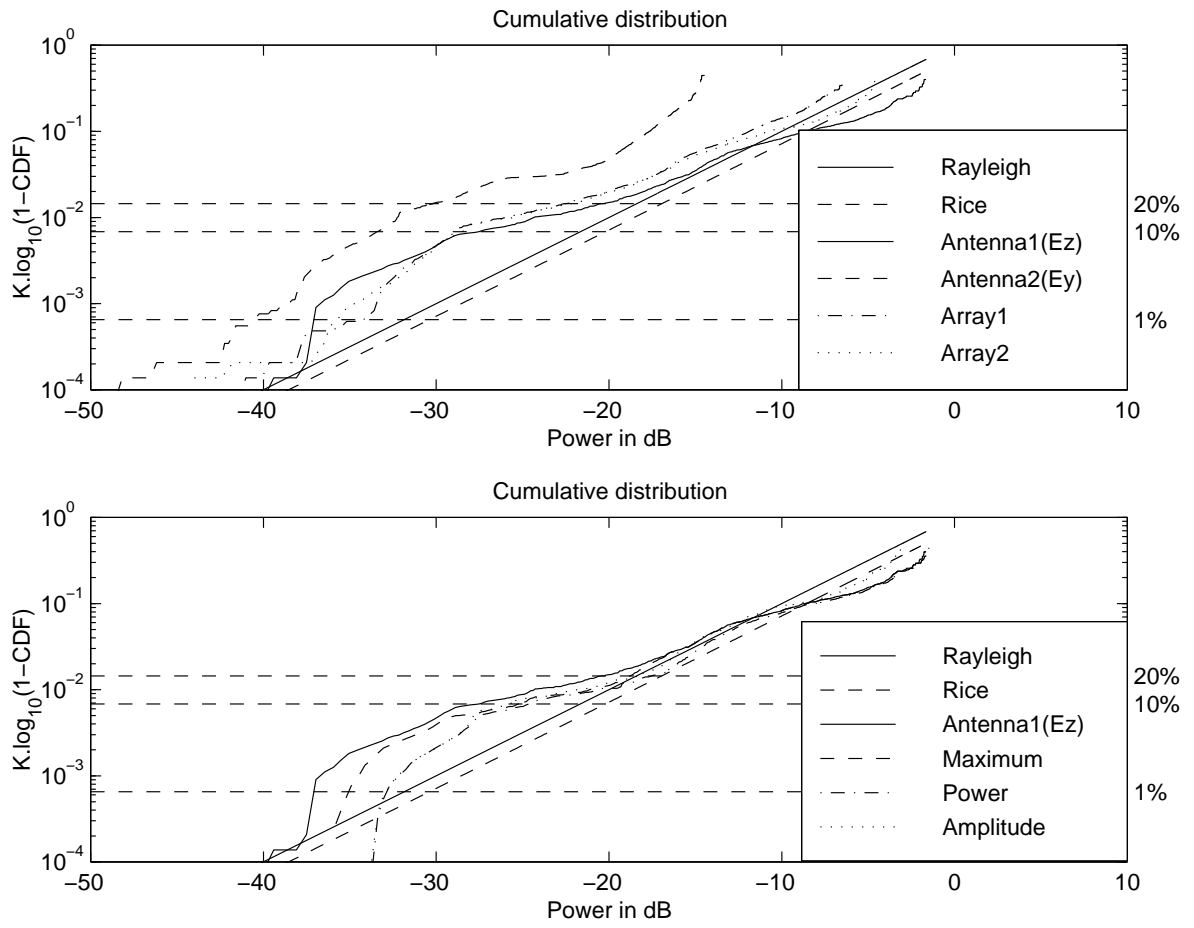

Fig. 7: Cumulative distribution functions of the combined antenna and polarisation diversity.

The 3-antenna case was giving of course nearly identical correlations as for the 2-antenna case, but with the correlation of the elements 1 and 3 being on a distance scale half of that of the 12 and 23 elements. The improvements are better than for the case of 2 antennas (of the order of $3 \mathrm{~dB}$, Fig. 8). This is not sufficient to justify the added complexity of the receiver. 

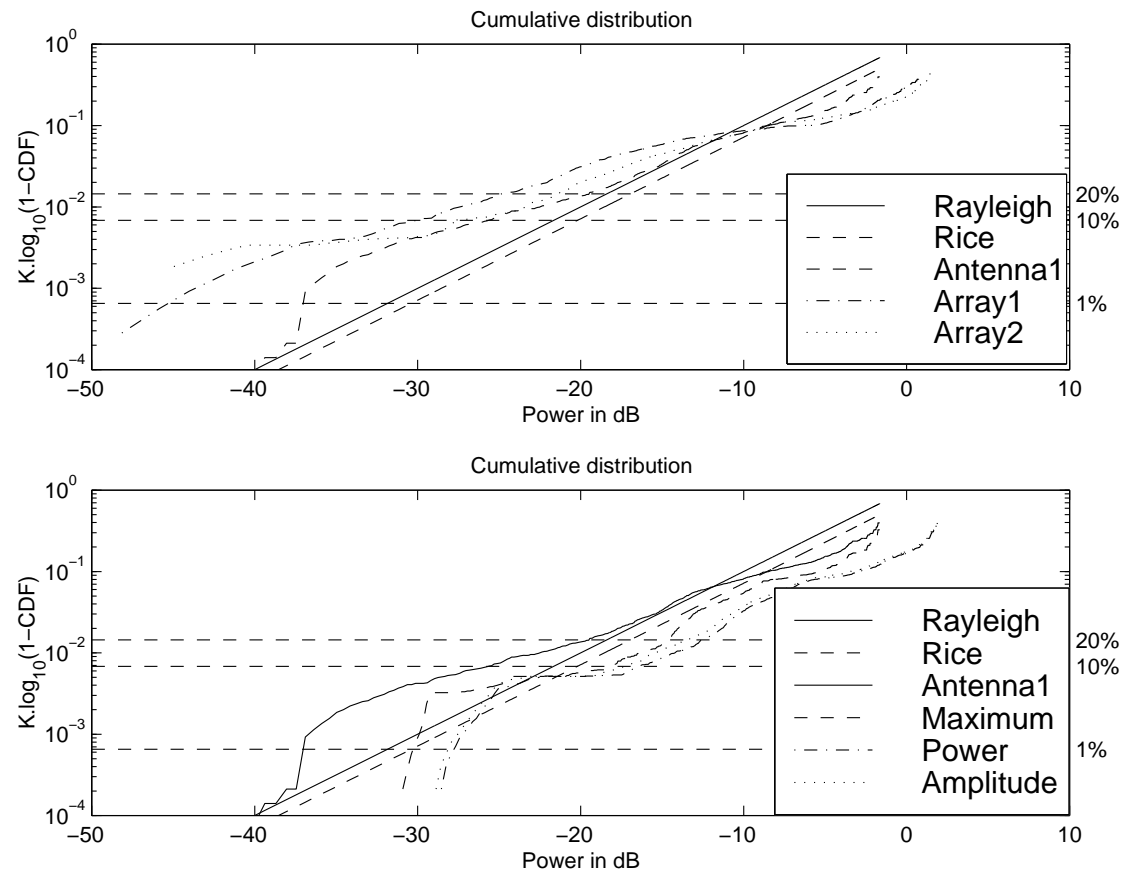

Fig. 8: Cumulative distribution functions of the 3-antenna diversity.

All results are summarised in Table 2, where the improvements of the signal levels with respect to the single antenna case for the 5 combination methods and the 4 diversity cases are recapitulated.

Table 2: Improvement with respect to 1 antenna in $99 \%$ of the cases.

\begin{tabular}{|l|r|r|r|r|r|}
\hline & \multicolumn{1}{|l|}{ Linear(1) } & \multicolumn{1}{|l|}{ Circular(2) } & \multicolumn{1}{l|}{ Maximum } & Amplitude & Power \\
\hline 2-ant. & -7.0 & -0.1 & 4.3 & 5.5 & 5.7 \\
\hline Pol. & -22.0 & -0.3 & 0.4 & 3.0 & 3.0 \\
\hline a\&p & 2.8 & 1.4 & 2.0 & 4.2 & 4.2 \\
\hline 3-ant. & -7.5 & -8.7 & 6.8 & 9.0 & 9.3 \\
\hline
\end{tabular}

We can conclude that in all cases the power combining method is the best, followed closely with amplitude and maximum combinations, and that antenna diversity is better than polarisation diversity. The figures of Table 2 may look high, but they are the effective improvements at system level. Indeed an improvement of the signal at places, where it is already acceptable, does not improve the coverage significantly, but it does where the level is sufficiently low.

\section{Acknowledgement:}

This research has been supported by the IT Programme of the Flemish Government (ISIS).

\section{References:}

[1] Rodney G. Vaughan \& J. Bach Andersen, Antenna Diversity in Mobile Communications, IEEE trans. on vehicular technology, Vol. VT-36, no. 4, November 1987

[2] I. De Coster, G. Anon Madariaga, B. Pazos Souto, E. Van Lil \& F. Pérez-Fontán, An Extended Propagation Software Package For Indoor Communication Systems, Proc. ICAP 1997, Edinburgh, UK, April 14-17, 1997, p.2.298-p.2.301

[3] Martin Steppler, ComNets Annual Report 1996, RWTH-Aachen

[4] Silvia Ruiz-Boqué \& Ramón Agustí Comes, Polarisation diversity for indoor mobile communications, COST 231 TD(93)

[5] ITU-R Recomm. nr. PN.1057, 1994 ITU; Radiocomm. sector, 1211 Geneva 20, Switserland 